\title{
Convergence of the Viscosity Method for a Nonstrictly Hyperbolic Conservation Law
}

\author{
Yunguang Lu \\ Wuhan Institute of Mathematical Sciences, Academia Sinica, P.O. Box 71007, \\ Wuhan 430071, PRC
}

Received December 17, 1991; in revised form June 29, 1992

Abstract. A convergence theorem for the method of artificial viscosity applied to the nonstrictly hyperbolic system $v_{t}+(v u)_{x}=0, u_{t}+\left(\frac{1}{2} u^{2}+\int^{v} s(s+\delta)^{r-3} d s\right)_{x}=0$ $(\delta>0, r>3)$ is established. Convergence of a subsequence in the strong topology is proved without uniform estimates on the derivatives using the theory of compensated compactness and an analysis of progressing entropy waves.

\section{Introduction}

In this paper we consider the existence of global weak solutions for nonlinear hyperbolic conservation laws

$$
\left\{\begin{array}{l}
v_{t}+(v u)_{x}=0 \\
u_{t}+\left(\frac{1}{2} u^{2}+\int^{v} s(s+\delta)^{r-3} d s\right)_{x}=0
\end{array}\right.
$$

with initial data

$$
(v(x, 0), u(x, 0))=\left(v_{0}(x), u_{0}(x)\right),
$$

where $\delta, r$ are positive constants and $r>3$. When $\delta=0,(1.1)$ is motivated by the isentropic equation of gas dynamics for a polytropic gas. The global weak solutions of which had been solved for the case of $1<r<3$ by using the Glimm difference scheme [1]. In the present paper, we shall study the system (1.1) with bounded measurable initial data (1.2) by using the established technique of compensated compactness given in [2,3]. Through an analysis of progressing entropy waves, we establish a convergence theorem for the method of artificial viscosity applied to the system (1.1) and obtain the existence of the global weak solutions for the Cauchy problem (1.1), (1.2). 
Let $F$ be the mapping from $E^{2}$ into $E^{2}$ defined by

$$
F:(v, u) \rightarrow\left(v u, \frac{1}{2} u^{2}+\int^{v} s(s+\delta)^{r-3} d s\right),
$$

then two eigenvalues of $d F$ are

$$
\lambda_{1}=u-v(v+\delta)^{\frac{1}{2}(r-3)}, \quad \lambda_{2}=u+v(v+\delta)^{\frac{1}{2}(r-3)}
$$

with corresponding right and left eigenvectors

$$
r_{1}=\left(1,-(v+\delta)^{\frac{1}{2}(r-3)}\right)^{T}, \quad r_{2}=\left(1,(v+\delta)^{\frac{1}{2}(r-3)}\right)^{T},
$$

and

$$
l_{1}=\left(1,-(v+\delta)^{\frac{1}{2}(r-3)}\right), \quad l_{2}=\left(1,(v+\delta)^{\frac{1}{2}(r-3)}\right) .
$$

Therefore, it follows from (1.3) that $\lambda_{1}=\lambda_{2}$ at line $v=0$ at which the strictly hyperbolicity fails to hold.

In this paper, we mainly obtain the following existence theorem.

Theorem 1.1 (Main theorem). Let the initial data $\left(v_{0}(x), u_{0}(x)\right)$ be bounded measurable and $v_{0}(x) \geqq 0$. Then there exist a subsequence $\left(v^{\varepsilon_{n}}(x, t), u^{\varepsilon_{n}}(x, t)\right)$ of the viscosity solutions $\left(v^{\varepsilon}(x, t), u^{\varepsilon}(x, t)\right)$ given by (2.1), (1.2) and the bounded measurable functions $v(x, t)$ and $u(x, t)(v(x, t) \geqq 0)$ such that

$$
v^{\varepsilon_{n}}(x, t) \rightarrow v(x, t), \quad u^{\varepsilon_{n}}(x, t) \rightarrow u(x, t), \quad \text { a.e. on } \Omega,
$$

where $\Omega \subset R^{*} R^{+}$is any bounded, open set. Therefore, $(v(x, t), u(x, t))$ is an admissible solution of the Cauchy problem (1.1), (1.2).

The program of this paper is as follows: In Sect. 2 we consider the existence of viscosity solutions for the system (1.1) which is based on a priori- $L^{\infty}$ estimate given by using the framework of positively invariant regions [4]. In Sect. 3 we construct four families of entropy-entropy flux of Lax type which we use to prove in Sect. 4 that the compactly probability measures $v$ are indeed Dirac ones.

\section{Viscosity Solutions}

In this section we consider the Cauchy problem for the related parabolic system

$$
\left\{\begin{array}{l}
v_{t}+(v u)_{x}=\varepsilon v_{x x} \\
u_{t}+\left(\frac{1}{2} u^{2}+\int^{v} s(s+\delta)^{\frac{1}{2}(r-3)} d s\right)_{x}=\varepsilon u_{x x}
\end{array}\right.
$$

with the initial data (1.2).

In the paper [4], the authors have given the existence of the solutions of the Cauchy problem (2.1),(1.2) when $\delta=0$. In their framework, the basic difficulty is to induce a priori- $L^{\infty}$ estimate on solutions.

By the general invariant regions Theorem 4.3 in [4], it is easy to get the following results:

Theorem 2.1 (A priori bounds theorem). If $v_{0}(x), u_{0}(x)$ are bounded measurable and $v_{0}(x) \geqq 0$, then

$$
\Sigma=\{(v, u): w(u, v) \leqq \text { const }, z(u, v) \geqq \text { const, } v \geqq 0\}
$$


Fig. 1

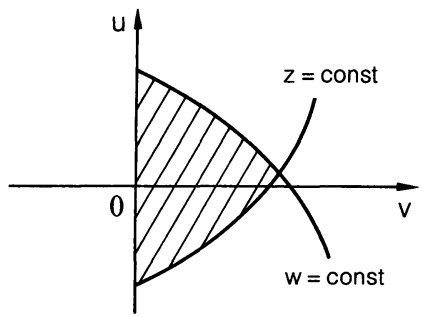

is invariant regions (Fig. 1), where $w=u+\frac{1}{2}(r-1)(v+\delta)^{\frac{1}{2}(r-1)}$ and $z=u-\frac{1}{2}(r-1)(v+\delta)^{\frac{1}{2}(r-1)}$ are two Riemann invariants of the system (1.1).

From Theorem 2.1, the solutions of the Cauchy problem (2.1), (1.2) have a priori- $L^{\infty}$ estimate

$$
0 \leqq v^{\varepsilon}(x, t) \leqq M, \quad\left|u^{\varepsilon}(x, t)\right| \leqq M .
$$

Here $M$ is a positive constant depending only on the initial data. Therefore, the following global existence of solutions is obtained.

Theorem 2.2. Let $v_{0}(x), u_{0}(x)$ be bounded measurable and $v_{0}(x) \geqq 0$. Then for any fixed $\varepsilon>0$, the Cauchy problem (2.1), (1.2) has a unique global solution $\left(v^{\varepsilon}(x, t), u^{\varepsilon}(x, t)\right)$ that satisfies (2.2).

Noticing that the system (1.1) has a strictly convex entropy $\eta=\frac{1}{(r-2)(r-1)}(v+\delta)^{r-1}+\frac{1}{2} u^{2}$, we deduce that $[5,6]$

$$
\varepsilon^{1 / 2} \partial_{x} v, \varepsilon^{1 / 2} \partial_{x} u \text { are uniformly bounded in } L_{\text {loc }}^{2}\left(R^{*} R^{+}\right) \text {. }
$$

From (2.3) and the boundedness of $\left(v^{\varepsilon}, u^{\varepsilon}\right)$, we have the following Theorem 2.3.

Theorem 2.3. For any $C^{2}$ entropy pairs $(\eta(v, u), q(v, u))$ of the system (1.1),

$$
\eta\left(v^{\varepsilon}, u^{\varepsilon}\right)_{t}+q\left(v^{\varepsilon}, u^{\varepsilon}\right)_{x} \text { is compact in } H_{\mathrm{loc}}^{-1}\left(R^{*} R^{+}\right) \text {. }
$$

Proof. It follows immediately from Theorem 3 in [10].

The above theorem guarantees (4.1) in Sect. 4 to be true. Equation (4.1) is the soul of the theory of compensated compactness and we will use it with the progressing entropy waves constructed in the next section to prove Theorem 1.1 in Sect. 4.

\section{Entropy Waves}

This section is concerned with entropy waves for the system (1.1). We recall that a pair of real-valued maps $(\eta, q)$ is an entropy for (1.1) if all smooth solutions satisfy

$$
\nabla \eta(v, u) \cdot \nabla F(v, u)=\nabla q(v, u) .
$$

We are going to introduce progressing waves for the entropy equations (3.1) in the method given by DiPerna in [3].

The entropy equations are equivalent to

$$
\eta_{v v}=(v+\delta)^{r-3} \eta_{u u}
$$


If $k$ denotes a constant, then the function $\eta=h(s) e^{k u}$ solved (3.2) provided

$$
h^{\prime \prime}(v)-k^{2}(v+\delta)^{r-3} h=0 .
$$

Equation (3.3) may be transformed into a standard Fuchsian equation using the change of variables $a(v)=(v+\delta)^{\frac{1}{4}(3-r)}, s=\frac{2 k}{r-1}(v+\delta)^{\frac{1}{2}(r-1)}$. Then $h=a(v) \varphi(s)$ solves (3.3) if and only if

$$
\varphi^{\prime \prime}-\left(1+\mu s^{-2}\right) \varphi=0,
$$

where $\mu=\left(4-(r-1)^{2}\right) / 4(r-1)^{2}>-\frac{1}{4}$. Using (3.1) we have

$$
q_{u}=v \eta_{v}+u \eta_{u}
$$

and a progressing wave of the system (1.1) is provided by $\eta=h(v) e^{k u}$, $q=u \eta+\left(v h^{\prime}-h\right) e^{k u} / k$. Let $h=a(v) \varphi(s)$, then

$$
q=\eta\left(u+v(v+\delta)^{\frac{1}{2}(r-3)} \varphi^{\prime} / \varphi-((r+1) v+4 \delta) / 4 k(v+\delta) .\right.
$$

We may use the method of Frobenius to give the solutions of the Fuchsian equation (3.4) with a series of the form $\varphi(s)=\sum_{n \geqq j} e_{n} s^{n}$.

Then two independent solutions of (3.4) are

$$
\varphi_{+}(s)=s^{j+} \sum_{n=0} e_{n} s^{n}, \quad \varphi_{-}(s)=s^{j-} \sum_{n=0} d_{n} s^{n},
$$

where $j_{+}, j_{-}$are two distinct roots of the equation $j(j-1)=\mu\left(j_{+}>j_{-}\right)$and

$$
c_{n}=\frac{c_{n-1}}{\left(2 n+j_{+}\right)\left(2 n+j_{-}-1\right)-\mu}, \quad d_{n}=\frac{d_{n-1}}{\left(2 n+j_{-}\right)\left(2 n+j_{-}-1\right)-\mu}, \quad n \geqq 1 .
$$

By using the comparison theorem [11], we have $\varphi_{-}(s)-\varphi_{+}(s)>0$ as $s>0$ for any given constants $c_{0}>0, d_{0}>0$. Moreover, we may especially choose positive constants $c_{0}, d_{0}$ such that $[11,3]$

$$
\left\{\begin{array}{l}
\varphi_{1} e^{-s}=1+O\left(s^{-2}\right), \quad \varphi_{1}^{\prime} / \varphi_{1}=1+O\left(s^{-2}\right), \\
\varphi_{2} e^{s}=1+O\left(s^{-2}\right), \quad \varphi_{2}^{\prime} / \varphi_{2}=-1+O\left(s^{-2}\right)
\end{array}\right.
$$

as $s$ approaches infinity, where $\varphi_{1}=\varphi_{-}, \varphi_{2}=\varphi_{-}-\varphi_{+}$.

Let $\eta_{k}^{1}=a(v) \varphi_{1} e^{k u}$, then

$$
\eta_{k}^{1}=a(v) \varphi_{1} e^{-s} e^{k w}=e^{k w}\left(a+O\left(k^{-2}\right)\right)
$$

on $v \geqq 0$ and the corresponding flux function is of the form

$$
\begin{aligned}
q_{k}^{1}= & \eta_{k}^{1}\left(u+v(v+\delta)^{\frac{1}{2}(r-3)}+v(v+\delta)^{\frac{1}{2}(r-3)}\left(\varphi_{1}^{\prime} / \varphi_{1}-1\right)\right. \\
& -((r+1) v+4 \delta) / 4 k(v+\delta)=\eta_{k}^{1}\left(\lambda_{2}+O\left(k^{-1}\right)\right)
\end{aligned}
$$

on $v \geqq 0$.

In a similar way one shows that the entropy

$$
\eta_{-k}^{1}=a(v) \varphi_{1} e^{-k u}=a \varphi_{1} e^{-s} e^{-k z}=e^{-k z}\left(a+O\left(k^{-2}\right)\right)
$$

on $v \geqq 0$ and the corresponding flux

$$
\begin{aligned}
q_{-k}^{1} & =\eta_{-k}^{1}\left(u-v(v+\delta)^{\frac{1}{2}(r-3)} \varphi_{1}^{\prime} / \varphi_{1}+\frac{(r+1) v+4 \delta}{4 k(v+\delta)}\right. \\
& =\eta_{-k}^{1}\left(\lambda_{1}+O\left(k^{-1}\right)\right)
\end{aligned}
$$


on $v \geqq 0$. The entropy pair $\left(\eta_{k}^{2}, q_{k}^{2}\right)$ satisfies

$$
\eta_{k}^{2}=a \varphi_{2} e^{-k u}=a \varphi_{2} e^{s} e^{k z}=e^{k z}\left(a+O\left(k^{-2}\right)\right)
$$

on $v \geqq 0$ and

$$
q_{k}^{2}=\eta_{k}^{2}\left(\lambda_{1}+O\left(k^{-1}\right)\right)
$$

on $v \geqq 0$. The entropy pair $\left(\eta_{-k}^{2}, q_{-k}^{2}\right)$ satisfies

$$
\eta_{-k}^{2}=a \varphi_{2} e^{-k u}=e^{-k w}\left(a+O\left(k^{-2}\right)\right), \quad q_{-k}^{2}=\eta_{-k}^{2}\left(\lambda_{2} O\left(k^{-1}\right)\right)
$$

on $v \geqq 0$.

If we further analyse the property of $\left(\zeta_{k}^{1}, q_{k}^{1}\right)$, we have from (3.8)-(3.10) that

$$
q_{k}^{1}=\eta_{k}^{1} \lambda_{2}-e^{k w}\left[((r+1) v+4 \delta) / 4 k(v+\delta)^{\frac{1}{4}(1+r)}+O\left(\frac{1}{k^{2}}\right)\right],
$$

and so

$$
\lambda_{2} \eta_{k}^{1}-q_{k}^{1}=e^{k w}\left[\left((v+\delta)^{\frac{1}{4}(3-r)}+\frac{1}{4}(r-3) v(v+\delta)^{-\frac{1}{4}(1+r)}\right) / k+O\left(\frac{1}{K^{2}}\right)\right] .
$$

The latter property (3.16) is basic to our analysis in the next section.

\section{The Proof of Theorem 1.1}

Consider a compactly supported probability measure $v$ on $R^{2}$ such that

$$
\left\langle v, \eta_{1}\right\rangle\left\langle v, q_{2}\right\rangle-\left\langle v, \eta_{2}\right\rangle\left\langle v, q_{1}\right\rangle=\left\langle v, \eta_{1} q_{2}-\eta_{2} q_{1}\right\rangle
$$

for all $C^{2}$ entropy pairs $\left(\eta_{i}, q_{i}\right)(i=1,2)$ of the system (1.1). Then the proof of Theorem 1.1 is reduced to prove that $v$ is a point mass by the well-known framework of the theory of compensated compactness.

Let $Q$ denotes the smallest characteristic rectangle

$$
Q=\left\{(v, u): w_{-} \leqq w(v, u) \leqq w_{+}, z_{-} \leqq z(v, u) \leqq z_{+}\right\} .
$$

As done in [2], we introduce probability measures $\mu_{k}^{ \pm}$on $Q$ defined by

$$
\left\langle\mu_{k}^{+}, h\right\rangle=\left\langle v, h \eta_{k}^{1}\right\rangle /\left\langle v, \eta_{k}^{1}\right\rangle
$$

and

$$
\left\langle\mu_{k}^{-}, h\right\rangle=\left\langle v, h \eta_{-k}^{2}\right\rangle\left\langle\left\langle v, \eta_{-k}^{2}\right\rangle,\right.
$$

where $h=h(v, u)$ denotes an arbitrary continuous function. As a consequence of weak-star compactness, there exist probability measures $\mu^{ \pm}$on $Q$ such that

$$
\left\langle\mu^{ \pm}, h\right\rangle=\lim _{k \rightarrow \infty}\left\langle\mu_{k}^{ \pm}, h\right\rangle
$$

after the selection of an appropriate subsequence. We observe that the measures $\mu^{+}$and $\mu^{-}$are respectively concentrated on the boundary sections of $Q$ associated with $w$, i.e.

$$
\operatorname{Qn}\left\{(v, u): w=w^{+}\right\} \text {and } Q n\left\{(v, u): w=w^{-}\right\}
$$


Pay attention to that $\left(\eta_{k}^{1}, q_{k}^{1}\right)$ and $\left(\eta_{-k}^{2}, q_{-k}^{2}\right)$ satisfy

$$
q_{k}^{1}=\eta_{k}^{1}\left(\lambda_{2}+O\left(k^{-1}\right)\right), \quad q_{-k}^{2}=\eta_{-k}^{2}\left(\lambda_{2}+O\left(k^{-1}\right)\right)
$$

on $v \geqq 0$, we have as given in [2] that

$$
\left\langle\mu^{+}, \lambda_{2} \eta-q\right\rangle=\left\langle\mu^{-}, \lambda_{2} \eta-q\right\rangle
$$

for any $C^{2}$ entropy pair $(\eta, q)$.

On account of

$$
\begin{aligned}
& \left\langle\mu^{-}, \lambda_{2} \eta_{k}^{1}-q_{k}^{1}\right\rangle \leqq c_{1} e^{k w^{-}} / k \\
& \left\langle\mu^{+}, \lambda_{2} \eta_{k}^{1}-q_{k}^{1}\right\rangle \geqq c_{2} e^{k w^{+}} / k
\end{aligned}
$$

from (3.16), where $c_{1}, c_{2}$ are positive constants, so (4.7) deduces that $w^{+}=w^{-}$from (4.6). In the same fashion we conclude that $z^{+}=z^{-}$. This completes the proof of Theorem 1.1.

\section{References}

1. DiPerna, R.J.: Global solutions to a class of nonlinear hyperbolic systems of equations. Comm. Pure Appl. Math. 26, 1-28 (1973)

2. DiPerna, R.J.: Convergence of approximate solutions to conservation laws. Arch. Rational Mech. Anal. 82, 27-70 (1983)

3. DiPerna, R.J.: Convergence of the viscosity method for isentropic gas dynamics. Commun. Math. Phys. 91, 1, 1-30 (1983)

4. Chueh, K.N., Conley, C.C., Smoller, J.A.: Positively invariant regions for systems of nonlinear diffusion equations. Ind. Univ. Math. J. 26, 372-411 (1977)

5. Lu, Y.: Cauchy problem for an extended model of combustion. Proceedings of the Royal Society of Edinburgh (to appear)

6. Lu, Y.: Cauchy problem for a hyperbolic model (to appear)

7. Lu, Y.: Convergence of solutions to nonlinear dispersive equations without convexity conditions. Applicable Analysis 31, 4: 239-246 (1989)

8. Lu, Y.: Convergence of the viscosity method for a nonstrictly hyperbolic system. Acta Mathematica Scientia, Vol. 12, No. 2 (1992)

9. Chen, G., Lu, Y.: The study on application way of the compensated compactness theory. Chinese Science Bulletin 34, 1: 15-19 (1989)

10. Ding, X., Chen, G., Luo, P.: Convergence of the Lax-Friedrichs scheme for isentropic gas dynamics. Acta Mathematica Sinica 4, 483-500 (1985)

11. Kamake, E.: Differentialgleichungen, 1971

Communicated by A. Jaffe 